\title{
Safety Effects of Connected and Automated Vehicle-Based Variable Speed Limit Control near Freeway Bottlenecks considering Driver's Heterogeneity
}

\author{
Ye Li, ${ }^{1}$ Yuntao Shi, ${ }^{1}$ Jaeyoung Lee, ${ }^{1}$ Chen Yuan $\mathbb{D}^{1,2}$ and Baojie Wang ${ }^{3}{ }^{3}$ \\ ${ }^{1}$ School of Traffic and Transportation Engineering, Central South University, Changsha, Hunan, China \\ ${ }^{2}$ Department of Computer Science, City University of Hong Kong, Hong Kong, China \\ ${ }^{3}$ Key Laboratory of Transport Industry of Management, Control and Cycle Repair Technology for Traffic Network \\ Facilities in Ecological Security Barrier Area, Chang'an University, Xi'an, China
}

Correspondence should be addressed to Baojie Wang; wangbj2@163.com

Received 5 October 2021; Revised 1 December 2021; Accepted 21 December 2021; Published 30 January 2022

Academic Editor: Arkatkar Shriniwas

Copyright (C) $2022 \mathrm{Ye} \mathrm{Li} \mathrm{et} \mathrm{al.} \mathrm{This} \mathrm{is} \mathrm{an} \mathrm{open} \mathrm{access} \mathrm{article} \mathrm{distributed} \mathrm{under} \mathrm{the} \mathrm{Creative} \mathrm{Commons} \mathrm{Attribution} \mathrm{License,} \mathrm{which}$ permits unrestricted use, distribution, and reproduction in any medium, provided the original work is properly cited.

\begin{abstract}
A control strategy using variable speed limit (VSL) is a proven solution to reduce freeway collision risks and improve safety. However, the heterogeneity of human drivers restricts the effectiveness of traditional VSL controls, which may be made up by recent advanced technologies of connected and automated vehicles (CAVs). This study aims to propose a CAV-based VSL control system to address the limitations caused by human drivers on VSL control's effectiveness. First, the heterogeneity of human drivers is analyzed, and its impact on the safety performance of VSL is examined. Specifically, a microscopic simulation platform is established, and two vehicle dynamic models developed for CAVs and human-driven vehicles (HDVs) are incorporated into the simulation platform. Based on a widely utilized surrogate safety measurement, time-tocollision, its derivative metrics are applied to evaluate collision risks, and the total travel time is used to assess operational efficiency. Extensive simulations are conducted to examine the performance of the proposed CAV-VSL system. The results indicate the following: (1) the heterogeneity of human drivers negatively affects the performance of the VSL; (2) the performance of the proposed control system in a mixed flow can be improved by advanced wireless communication technology; (3) CAVs are able to implement the VSL control strategy effectively resulting in the proactive reduction of the heterogeneity.
\end{abstract}

\section{Introduction}

Freeway bottlenecks have attracted considerable attention from transportation researchers since the bottlenecks drastically reduce both efficiency and safety [1-5]. The formation of traffic bottlenecks is caused by a variety of reasons, such as lane capacity reduction, temporary lane closure, and crashes. The speed of vehicles downstream reduces when approaching the bottleneck area and a shockwave is propagated to the upstream, which increases rear-end collision risks of upstream vehicles. In order to mitigate the rear-end collision risks caused by freeway bottlenecks, various safety management countermeasures have been implemented in practice, such as variable speed limit (VSL) control, ramp metering, managed lanes dynamic routing, and hard shoulder running [2, 4-11].

The VSL control is a proven countermeasure in practice to improve safety near freeway bottlenecks $[2,4,7,8,10-12]$. The core idea of VSL is to proactively adjust vehicles' speeds upstream and reduce the speed difference of vehicles between the upstream and downstream parts, so as to decrease rear-end collision risks $[13,14]$. The previously proposed VSL control strategies can be classified into two main groups: feedback-based controllers and prediction-based controllers. Details could be referred to in the section of the literature review.

However, the practical effectiveness of VSL control is restricted by human drivers' heterogeneity [7, 15-17]. For 
example, the different sight distances of drivers result in inconsistent drivers' reactions to the variable speed limit provided by a variable message sign (VMS). When observing the speed limit, different drivers might also have disparate performances. Some drivers do not comply with the variable speed limit, while other conservative drivers have an overcompliance behavior and reduce to an excessively low speed. The abovementioned heterogeneity issues may negatively impact the performances of VSL control strategies in practice.

With the recent advancement of communication and automation technologies, connected and automated vehicles (CAVs) have been developed. The CAVs might be capable of enhancing the performance of VSL control. The advanced vehicle-to-vehicle and vehicle-to-infrastructure communication technologies provide the information-exchange environment, so vehicles could acquire downstream traffic information beyond the perception field of human drivers. Meanwhile, the automation technique allows vehicles to operate at the accurate speed required by VSL control. Through real-time and accurate information exchange and intelligent vehicle control, the heterogenous responses to VSL control might be avoided. Furthermore, even considering the mixed traffic flow consisting of CAVs and humandriven vehicles (HDVs), the collaboration of VSL into CAVs might be also effective. The CAVs at the downstream decelerate accurately in advance according to the required speed limit, and then, the following HDVs will be forced to slow down to keep a safe car-following speed. When the market penetration rate (MPR) of CAVs reaches a certain point, the driving heterogeneity of human drivers would be reduced, as well as the speed variations.

Although some studies have integrated VSL with connected vehicles to reduce rear-end collision risks $[7,8,17-20]$, the above heterogeneity issues have not been fully explored yet. Therefore, the main objective of this study is to propose a CAV-VSL integrated control system to reduce rear-end collision risks near freeway bottlenecks considering the heterogeneity of human drivers. A microscopic simulation platform is firstly developed, in which the intelligent driver model (IDM) model is used to capture the driving behaviors of both HDVs and CAVs. Then, the conceptual CAV-VSL feedback control system is proposed based on the occurrence conditions of rear-end collisions. A rear-end collision risk is evaluated based on time-to-collision (TTC), time exposed TTC (TET), and time-integrated TTC (TIT) measures. Simulation experiments are conducted to evaluate the safety improvement performance of the proposed control system in various scenarios, taking different MPRs of CAVs, the heterogeneity of human drivers, and the influence of CAV strategy execution site into account.

The major contributions of this study are two aspects: (1) the safety effects of human drivers' heterogeneity on VSL controls are examined; (2) a CAV-VSL integrated control system is proposed to reduce collision risks considering the heterogeneity of human drivers. The rest of this paper is organized as follows. The literature review is presented in Section 2. In Section 3, human factors affecting VSL control strategies are elaborated. Then, the CAV-VSL control system and algorithm are proposed in Section 4. The simulation platform is introduced in Section 5. Simulation results and conclusions are presented in Section 6 and Section 7, respectively.

\section{Literature Review}

Early VSL studies are mainly formulated on the basis of the simple feedback control logic. In such approaches, strategies are adjusted depending on variances of macroscopic traffic flow parameters, such as traffic volume, density, and mean speed, so as to harmonize speed differences and stabilize traffic flow. The representative feedback-based VSL control strategies were proposed by Carlson et al. and Iordanidou et al. [21, 22]. The advantages of the feedback-based control strategy are simple and easy to be implemented. When traffic conditions are not dramatically changed, it would provide satisfactory control effectiveness. However, the feedbackbased control strategy adjusts its control strategy according to the response of traffic conditions, so the hysteresis is inevitable. In turbulent traffic conditions, a frequent adjustment may produce a secondary disturbance and aggravate an instability of traffic flow. The controller may not be able to adjust in time to maintain the desired traffic condition under this circumstance and may lose the best control opportunity and cause some oscillations during the feedback cycle [23].

Another type of the VSL control approach is model predictive control (MPC), which can predict the trend of traffic flow evolution utilizing the traffic flow model and adopt control in advance to prevent the formation of traffic congestion. For example, Hegyi et al. applied the MPC to optimally coordinate VSL aiming at suppressing shockwaves [24]. The results showed that the coordination of speed limits eliminated the shock wave from the downstream and decreased the total travel time by $17.3 \%$. Wang and Ioannou developed a new second-order traffic model incorporating the effect of VSL and verified its validation by comparing simulation results with VISSIM microscopic simulation data [25]. Hadiuzzaman developed a cell transmission modelbased VSL control with the goal of improving capacity [26]. The simulation result showed that the implementation of VSL can reduce total travel time by $15 \%$ and increase traffic flow capacity by $7 \%$. A limitation of prediction-based controllers lies in the accuracy of short-term traffic prediction. Li et al. proposed a VSL control method based on the crash risk prediction model, which can be regarded as a special MPC control strategy [27]. The results showed that the combined control strategy successfully decreased the collision risks by $22.62 \%$ and reduced the injury severity of crashes by $14.67 \%$.

With the advancement of communication and automation technologies, there are also some studies that try to seek solutions to the traffic issues near the freeway bottleneck by integrating VSL control strategies and the advanced technologies of CAVs. Wu et al. developed a VSL control strategy, which aims to reduce the rear-end crash risk at freeway bottlenecks under fog conditions, and the strategy 
was tested in the fully connected vehicles (CV) environment [17]. Han et al. applied the connected vehicle (CV) technology and proposed three VSL strategies to improve bottleneck discharge rates and reduce system delays [18].

Although a majority of the studies showed that a VSL control has a significant effect on improving traffic safety $[13,28,29]$, field tests failed to achieve similar performances of the simulation experiments, possibly due to the high compliance assumption in the simulation studies [7, 30]. A driver's sight distance and noncompliance and overcompliance behaviors contribute to the variances of driver responses. Aggressive drivers will not fully follow the advised speed limit, while conservative drivers tend to slow down the speed below the specified speed. Li et al. pointed out that when drivers exceed the speed limit of just $5 \mathrm{mph}$ during the heavy rain, the safety improvement of VSL would be reduced by $6 \%$ [31]. The limited sight distance of a driver also has a negative impact on the effectiveness of the VSL control. If drivers cannot observe the posted speed limit posted timely in inclement weather, it would deteriorate the implementation of the control strategy $[17,32]$.

\section{Factors Affecting VSL Controls}

Driver's various responses to the speed limit have direct effects on the performance of VSL controls [7, 17, 31, 33]. Reducing the speed limit at the cost of efficiency may not achieve the desired safety improvements, since an extreme low-speed limit would make drivers resist to comply [14]. The improper responses of drivers to the speed limit can be summarized into two aspects, i.e., noncompliance and overcompliance. Noncompliance mainly refers to that the driver will not fully comply with the suggested speed when observing the speed limit posted on the VMS, resulting in a higher driving speed than the speed limit. On the other hand, conservative drivers tend to slow down below the recommended speed limit, which would cause small shock waves as well due to the overcompliance behaviors. In addition, the performance of VSL control is also affected by the human driver's sight distance. The driver's visual observation on VMS is the basis of the VSL strategy implementation. A longer sight distance allows a longer deceleration distance, leading to a more moderate deceleration behavior.

The heterogeneities can come from multiple aspects, such as different psychological and physiological characteristics of drivers. In this study, however, heterogeneities mainly consider sight distance, noncompliance, and overcompliance. The reasons include the following: (1) it is difficult to reveal psychological and physiological characteristics directly, so driving behaviors become alternative for heterogeneity analysis; (2) for VSL controls, previous studies have indicated that heterogenous sight distances and compliance behaviors significantly affect the control effectiveness. Therefore, this study takes these heterogeneities into account when developing the CAV-VSL integrated control system. This section expatiates the factors affecting the VSL controls and provides the basis for simulation experiments.
3.1. Sight Distance. The driver's sight distance considered in this study refers to the distance between the position of the driver when he or she perceives the speed limit and the position of VMS. The sight distance determines the deceleration distance for the driver to conduct the deceleration task and indirectly affects the deceleration rate. Figure 1 displays the basic control segment of VSL systems, which includes both detector and VMS. When vehicles approach the VMS, drivers recognize the suggested speed limit in the position $d$ meters away from the VMS, where $d$ represents the driver's sight distance. The detectors collect vehicle information of traffic flow and provide data input for VSL control systems.

3.2. Noncompliance Rate. Noncompliance refers to that a driver does not fully comply with the recommended speed. The noncompliance directly brings about a larger speed variance and significantly increases a rear-end collision risk [7, 34-36]. As shown in Figure 1, the driver recognizes the suggested speed limit $V_{v s l}$ at the position $d$ ahead of the VMS and starts to decelerate from the current speed $V_{\text {initial }}$ to the target speed $V_{v s l}$. It means that the driver's deceleration task is $\left(V_{\text {initial }}-V_{v s l}\right)$. However, the noncompliant driver only completes the deceleration task of $\left(V_{\text {initial }}-V_{v s l}\right) *\left(1-\mathrm{NC}_{\text {rate }}\right)$, due to the disobedience, where $\mathrm{NC}_{\text {rate }}$ represents the noncompliance rate. The actual target speed $V_{v s l}^{*}$ is calculated according to (1). To avoid the impact on safety during the deceleration execution stage, it is assumed that the driver decelerates dynamically in accordance with (2), where $V_{\text {current }}$ represents the speed at the current step and dis current $_{\text {represents the distance between }}$ the vehicle and the detector downstream.

$$
\begin{aligned}
V_{v s l}^{*} & =V_{\text {initial }}-\left(V_{\text {initial }}-V_{v s l}\right) *\left(1-\mathrm{NC}_{\text {rate }}\right), \\
\operatorname{dec} & =\frac{\left(V_{\text {current }}^{2}-\left(V_{v s l}^{*}\right)\right)^{2}}{2 * \operatorname{dis}_{\text {current }}} .
\end{aligned}
$$

3.3. Overcompliance Rate. In contrast to the noncompliance driving behavior, excessive enforcement of a speed limit is defined as an overcompliance driving behavior. Although the driver's overcompliance slightly reduces the average speed of the roadway, it may increase the speed variance. Specifically, we assume that a driver completes the deceleration task of $\left(V_{\text {initial }}-V_{v s l}\right) *\left(1+\mathrm{OC}_{\text {rate }}\right)$, where $\mathrm{OC}_{\text {rate }}$ represents the driver's overcompliance rate, as shown in equation (3). Other settings are the same as in equation (2).

Equation (4) considers the abovementioned heterogeneities from both noncompliance and overcompliance. Furthermore, considering the difference of imprecise speed perception of human drivers, we set the upper limit of noncompliance and overcompliance rate for drivers in simulation experiments, which confines the fluctuation of individual heterogeneity to a certain range from $0 \%$ to the preset upper limit with the uniform distribution, as shown in equations (5)-(6). $\mathrm{NC}_{\text {uplimit }}$ and $\mathrm{OC}_{\text {uplimit }}$ means the upper limit of $\mathrm{NC}_{\text {rate }}$ and $\mathrm{OC}_{\text {rate }}$, respectively. 


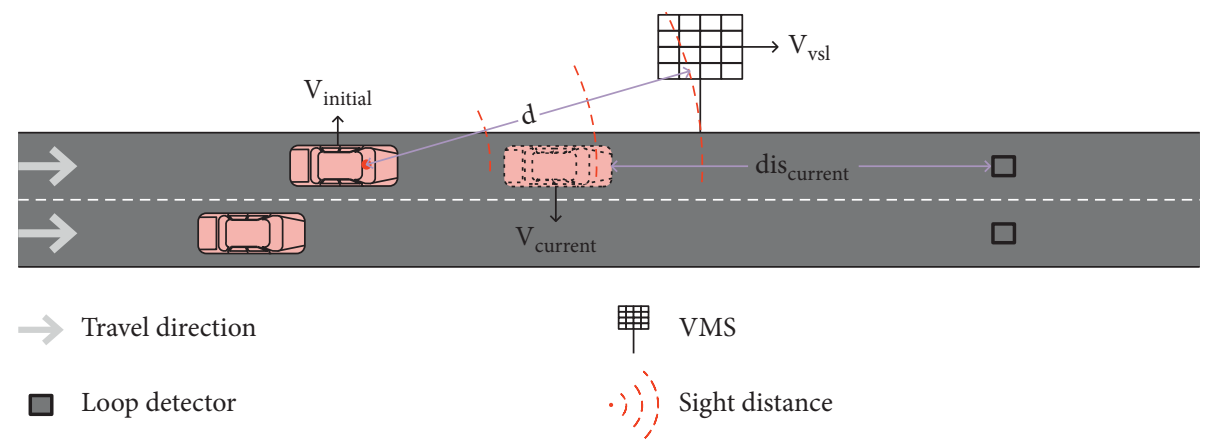

FIgURE 1: Illustration of the basic VSL control segment.

$$
\begin{aligned}
& V_{v s l}^{*}=V_{\text {initial }}-\left(V_{\text {initial }}-V_{v s l}\right) *\left(1+\mathrm{OC}_{\text {rate }}\right) \\
& V_{v s l}^{*}=V_{\text {initial }}-\left(V_{\text {initial }}-V_{v s l}\right) *\left(1-\mathrm{NC}_{\text {rate }}+\mathrm{OC}_{\text {rate }}\right)
\end{aligned}
$$$$
\mathrm{NC}_{\text {rate }}=\text { random.uniform }\left(0, \mathrm{NC}_{\text {uplimit }}\right),
$$$$
\mathrm{OC}_{\text {rate }}=\text { random.uniform }\left(0, \mathrm{OC}_{\text {uplimit }}\right) \text {. }
$$

\section{Development of the CAV-VSL Control System}

4.1. Control System Framework. The conceptual design of the proposed CAV-VSL control system is shown in Figure 2. The proposed system is constituted of data acquisition, central control, information release, and CAV unit modules. Among them, the CAV unit module includes information reception and onboard control modules. The operation process of the whole system is explained as follows:

Step 1: data acquisition: the traffic data collected by loop detectors, including traffic flow data (volume, density, and speed of both HDVs and CAVs), are sent to the central control module. The trajectory data of CAVs are also collected via wireless communication, including the position and speed of an individual vehicle.

Step 2: data processing: the central control module calculates the corresponding speed limit value based on the core algorithm with the collected data.

Step 3: information release: the central control module exchanges information with VMS (speed limit) and CAVs (speed limit and recommended strategy execution site) within the communication range. The VMS posts speed limit value after receiving it. The HDVs observe the VMS and response to the speed limit, while CAVs receive the precise speed limit via wireless communication.

Step 4: strategy implementation: the CAV calculates the optimal acceleration dynamically and adjusts the driving strategy through the onboard control module. The HDVs drive according to VSL control as well as the preceding CAVs' impact.
CAVs initiate the VSL control strategy when arriving in the recommended strategy execution site, while HDVs only adopt the conventional "observe and execute" manner. In the mixed traffic flow, the preceding CAVs could decelerate earlier than the following HDVs and enforce the HDVs to obey speed limit indirectly. The human driver's risky deceleration behavior and disobedience may be restrained to some extent.

Besides, CAVs with wireless communication functionality can accurately receive the speed limit information in advance regardless of the visibility limitation, which allows the VSL control execution site to change flexibly. It can also eliminate the defect that the human driver cannot observe the speed limit information timely during inclement weather. The automated driving technology ensures CAVs strictly and accurately decelerate according to the prescribed speed limit and eliminate the risk of traffic flow disturbance caused by the noncompliance and overcompliance of manual driving. Furthermore, receiving speed limit information in advance and implementing the deceleration beforehand can effectively increase the deceleration distance compared to the "observe and execute" manner. Vehicles with beforehand VSL information can achieve a moderate deceleration and it could help reduce the traffic flow fluctuation.

4.2. Core Control Algorithms. The implementation of VSL depends on whether there are frequent crashes, traffic congestion, or bottlenecks determined by historical data. In this study, the freeway segment with a bottleneck is hypothesized, as shown in Figure 2. There are two main triggering conditions for the proposed CAV-VSL control system. The first one is that the average speed difference between the upstream and downstream detectors exceeds $15 \mathrm{~km} / \mathrm{h}$ in the bottleneck area. The second is that the average speed difference of CAVs in two adjacent basic segments separated by detectors exceeds $15 \mathrm{~km} / \mathrm{h}$. When the control system is activated, two core control algorithms are adopted, including the central control module algorithm (Algorithm 1) and CAV onboard control module algorithm (Algorithm 2).

As shown in Algorithm 1, the central control module provides the update of the VSL control strategy. Previous studies have proposed a number of VSL control algorithms 


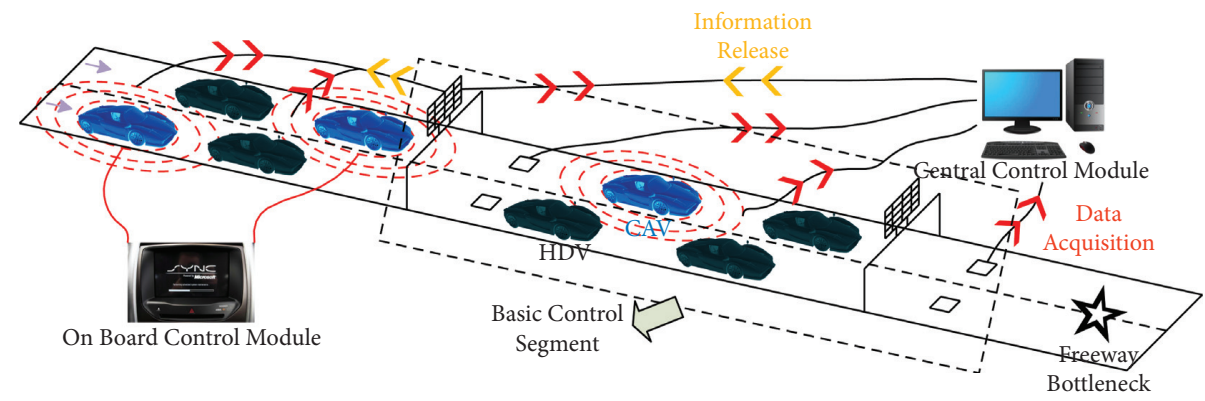

Figure 2: Conceptualization of the proposed CAV-VSL control system.

Input: The total simulation time, $T_{t}$; the number of detectors, $N$; the control cycle, $\Delta T$; the average speed collected from loop detector station at the location $x_{i+1}$ at time $t, V\left(x_{i+1}, t\right)$; the desired deceleration, $\beta$; the perception reaction time, $t_{a}$; the average length of the vehicles, $\bar{L}$; the average occupancy at location $x_{i}$ at time $t, O\left(x_{i}, t\right)$.

Output: The calculated speed limit, $V_{v s l}\left(x_{i}, t+\Delta T\right)$;

(1) for each $t \in\left[1, T_{t}\right]$ do

(2) if $\bmod (t, \Delta T)=0$ then

(3) $\quad i=\mathrm{N}-1$

(4) while $i>=1$ do

$$
\begin{aligned}
& \text { while } i>=1 \text { do } \\
& \qquad V_{v s l}\left(x_{i}, t+\Delta T\right) \longleftarrow V\left(x_{i+1}, t\right)-\beta * t_{a}+\operatorname{sqrt}\left(\beta * t_{a}\right)^{2}+2 * \beta * \bar{L} *\left(1-O\left(x_{i}, t\right) / O\left(x_{i}, t\right)\right)
\end{aligned}
$$$$
\text { return } V_{v s l}\left(x_{i}, t+\Delta T\right)
$$$$
i=i-1
$$

Algorithm 1: Central control module algorithm.

Input: The distance between CAV strategy execution site and the position of downstream VMS, $D_{C A V}$; the maximum acceleration, $\alpha_{m}$; the desired speed of subject vehicle, $v_{0}$; the minimum gap distance at a standstill, $s_{0}$; the gap distance between the subject and preceding vehicles, $s$; the safe time gap, $T$; the desired deceleration, $\beta$; the current speed of subject vehicle, $v$; the speed limit, $V_{v s l}$; the distance between subject vehicle and the detector at downstream, dis; the speed difference between subject and preceding vehicles, $\Delta v$; the average vehicle length, $\bar{L}$.

Output: The execution deceleration, $a$;

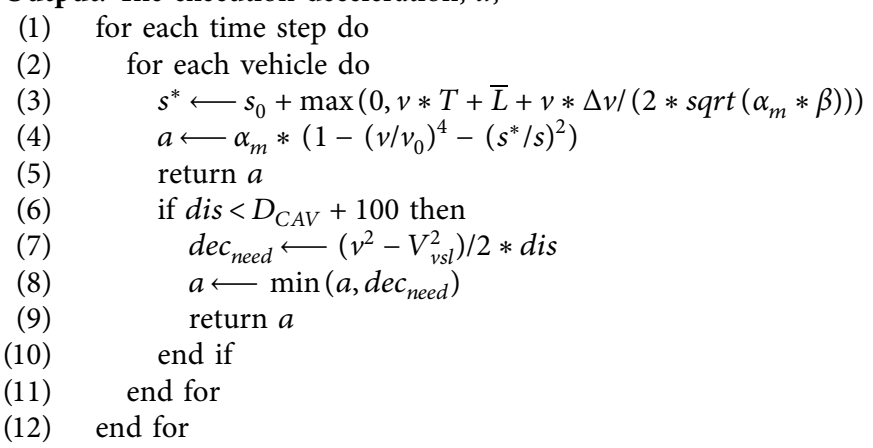

Algorithm 2: CAV on-board control module algorithm.

for different purposes, which can be divided into two categories: reducing collision risk and improving traffic efficiency $[2,5,7,10,11,31]$. In this study, the control algorithm was developed derived from our previous study whose control logic is based on the reduction of rear-end collision risk [31]. The basic theory of variable speed limit control is derived from the microscopic car-following scenario. Suppose that there are two consecutive vehicles on the freeway, and the following vehicle traveling at a high speed observes the leading vehicle traveling at a low speed. After a 
perception reaction time, the following vehicle starts to brake with the desired deceleration. Given the speed of the front vehicle and the gap distance between the two vehicles, the collision avoidance speed of the rear vehicle can be calculated. The upstream speed limit considering rear-end collision avoidance can be obtained by replacing the microscopic trajectory data with the aggregated traffic data collected by the loop detectors. For each VMS, an optimal speed limit can be first calculated by

$$
V_{v s l}\left(x_{i}, t+\Delta T\right)=V\left(x_{i+1}, t\right)-\beta t_{a}+\sqrt{\beta^{2} t_{a}^{2}+2 \beta \bar{L}\left[\frac{1-O\left(x_{i}, t\right)}{O\left(x_{i}, t\right)}\right]},
$$

where $V_{v s l}\left(x_{i}, t+\Delta T\right)$ represents the calculated speed limit to avoid the occurrence of rear-end collision at the location $x_{i}$ at time $t+\Delta T . \Delta T$ refers to the data aggregation interval of the loop detector. $V\left(x_{i+1}, t\right)$ represents the average speed collected from the loop detector station at the downstream location $x_{i+1}$ at time $t . t_{a}$ is the perception reaction time. $O\left(x_{i}, t\right)$ denotes the average occupancy at the location $x_{i}$ at the timestamp t. $\beta$ represents the desired deceleration of drivers. $\bar{L}$ means the average length of the vehicles.

The speed limit value $V_{v s l}\left(x_{i}, t+\Delta T\right)$ is calculated from downstream to upstream consecutively, but the calculated speed limit value cannot be posted on the VMS directly to avoid the turbulence of traffic flow. If the difference between the speed limits posted in two consecutive strategy execution periods is too large, the vehicles in the subsection would slow down sharply, forming a shock wave propagating back to upstream, which may increase the rear-end collision risks. Meanwhile, variable speed limit control is a gradual deceleration strategy. If the difference of the speed limit values posted in two consecutive VMSs is too large, it would also cause an oscillation of traffic flow. To solve the above issue, we further apply spatial-temporal constraints to the speed limit values, which can be calculated by

$$
\begin{aligned}
& V_{v s l}\left(x_{i}, t+\Delta T\right)=\max \left(\min \left(V_{v s l}\left(x_{i}, t+\Delta T\right), V_{v s l}\left(x_{i}, t\right)+\Delta_{v s l}\right), V_{v s l}\left(x_{i}, t\right)-\Delta_{v s l}\right), \\
& V_{v s l}\left(x_{i}, t+\Delta T\right)=\max \left(\min \left(V_{v s l}\left(x_{i}, t+\Delta T\right), V_{v s l}\left(x_{i+1}, t+\Delta T\right)+\Delta_{v s l}\right), V_{v s l}\left(x_{i+1}, t+\Delta T\right)-\Delta_{v s l}\right),
\end{aligned}
$$

where $V_{v s l}\left(x_{i}, t\right)$ represents the calculated speed of avoiding the occurrence of rear-end collision at the location $x_{i}$ at time t. $\Delta_{v s l}$ is the constraint range, which is set to be $15 \mathrm{~km} / \mathrm{h}$.

Another is the CAV onboard control module algorithm 2, which depends on the connected and automated functionalities of CAVs. The core idea of the algorithm is to flexibly change the execution site of CAV utilizing wireless communication and to achieve accurate dynamic deceleration relying on the automated function. All CAVs can receive the speed limit information beforehand, which makes CAVs adjust driving behaviors in advance to guide the following HDVs through interaction between vehicles. CAVs will receive speed limit information and recommended strategy execution sites within the communication range. After reaching the strategy execution site, CAVs compare the carfollowing acceleration calculated through the surrounding environment with the dynamic acceleration derived from the speed limit and position information and then adopt the smaller one to ensure a safe car-following distance while implementing the VSL control strategy.

\section{Simulation Platform}

5.1. Simulation Model. This study focuses on the exploration of the safety impact of human drivers' heterogeneity in the mixed traffic flow consisting of both HDVs and CAVs. One microscopic vehicle dynamic model, the intelligent driver model (IDM), is used to model the carfollowing behavior, which could capture the trajectory of individual vehicles and rear-end collisions risk arising from the interaction between vehicles. Notably, the authenticity of IDM has been proved for accurately reflecting human driving behavior [37-41]. The model calculates dynamic acceleration using desired speed and gap distance and can be expressed as follows:

$$
\begin{aligned}
& a=\alpha_{m}\left[1-\left(\frac{v}{v_{0}}\right)^{4}-\left(\frac{s^{*}}{s}\right)^{2}\right], \\
& s^{*}=s_{0}+\max \left[0, v T+\bar{L}+\frac{v \Delta v}{2 \sqrt{\alpha_{m} \beta}}\right],
\end{aligned}
$$

where $a$ denotes the acceleration of subject vehicle; $\alpha_{m}$ represents the maximum acceleration; $v$ and $v_{0}$ are the current speed and the desired speed of the subject vehicle, respectively; $s$ is the gap distance between the subject and preceding vehicles; $s_{0}$ represents the minimum gap distance at standstill; $T$ is the safe time gap; $\bar{L}$ is the average vehicle length; $\Delta v$ denotes the speed difference between subject and preceding vehicles; $\beta$ is the desired deceleration.

With the dynamic acceleration, vehicles' speeds and positions can be calculated as follows:

$$
\begin{aligned}
& v=v_{\text {prev }}+a \Delta t \\
& x=x_{\text {prev }}+v \Delta t+a \frac{(\Delta t)^{2}}{2},
\end{aligned}
$$

where $x_{\text {prev }}$ and $v_{\text {prev }}$ denote the position and speed of the subject vehicle in the previous time step; $\Delta t$ denotes the simulation time step.

Considering the real CAV model is publicly unavailable, many studies utilize the IDM to simulate dynamic behaviors of CAVs [38, 42-45]. The modified IDM model can be used to characterize the longitudinal dynamic behavior of CAVs. In this study, the reasons for applying the intelligent driver model (IDM) to describe dynamic behaviors of both human- 
driven vehicles and CAVs are as follows: (1) the real CAV model is not publicly available due to commercial confidentiality; (2) if different car-following model formulas are employed, the simulation results differences may result from different models instead of control strategies, which negatively affect the comparison of control performances; and (3) the IDM has been extensively utilized in previous studies to describe both human-driven vehicles and CAVs. In this study, the major differences considered between HDVs and CAVs are time gap and reaction time in the IDM, in which CAVs have the shorter time gap and response time. Details of parameter settings are described in the following subsection, and the same model provides a fair evaluation basis in simulation experiments.

5.2. Simulation Roadway. As shown in Figure 3, a simulated $10 \mathrm{~km}$ two-lane basic freeway roadway with a bottleneck was built via MATLAB 2019b software. The freeway roadway is divided into ten basic segments by detectors spaced one kilometer apart. Along this roadway, nine VMSs are deployed according to the position of the nine detectors upstream, while the most downstream detector is only used for the collection of traffic flow data. A low travel speed is set between detector 9 and detector 10 to simulate the "bottleneck" situation in simulation experiments. The simulated bottleneck can be caused by various incidents, such as crashes, lane closures, etc., with the common feature of low travel speed.

5.3. Evaluation Measurements. To evaluate the safety performance of the proposed CAV-VSL control system, appropriate surrogate safety measures, which quantifies the rear-end collision risk, should be applied. Surrogate safety measures bridge the gap between vehicle behaviors and potential traffic conflicts. Previous studies have proposed a variety of measurements for collision risk evaluation [46]. In this study, extended from the TTC index, time-exposed TTC (TET) and time-integrated TTC (TIT) measures were employed for the crash risk analysis. The TTC denotes the remaining time for the following vehicle to collide with the leading vehicle if they do not change driving states (change speeds or lanes). The TTC of the following vehicle $i$ at time step $t$ with respect to the leading vehicle $i-1$ can be calculated as follows:

$$
\operatorname{TTC}_{i}(t)= \begin{cases}\frac{x_{i-1}(t)-x_{i}(t)-L_{i-1}}{v_{i}(t)-v_{i-1}(t)}, & \text { if } v_{i}(t)>v_{i-1}(t), \\ \infty, & \text { if } v_{i}(t) \leq v_{i-1}(t),\end{cases}
$$

where $x_{i-1}$ and $v_{i-1}$ denote the position and speed of the preceding vehicle, respectively; $x_{i}$ and $v_{i}$ represent the position and speed of the following vehicle, respectively; and $L_{i-1}$ is the preceding vehicle's length.

According to the definition of the TTC index, the TTC value varies at each timestamp. Thus, the aggregated TET and TIT were utilized to represent the time aggregated crash risk, which can be calculated as

$$
\begin{aligned}
\operatorname{TET}(t) & =\sum_{i=1}^{M} \delta_{t} \Delta \Delta t, \delta_{t}= \begin{cases}1, & \forall 0<\operatorname{TTC}_{i}(t) \leq \mathrm{TTC}^{*}, \\
0, & \text { else, }\end{cases} \\
\operatorname{TET} & =\sum_{t=1}^{T I} \operatorname{TET}(t), \\
\operatorname{TIT}(t) & =\sum_{i=1}^{M}\left[\operatorname{TTC}^{*}-\operatorname{TTC}_{i}(t)\right] \Delta \Delta t, \forall 0<\operatorname{TTC}_{i}(t) \leq \mathrm{TTC}^{*}, \\
\operatorname{TIT} & =\sum_{t=1}^{T I} \operatorname{TIT}(t),
\end{aligned}
$$

where $\delta_{t}$ represents the switching variable at time $t ; \Delta t$ is the time step; $M$ is the number of vehicles; TI is time interval; TTC* denotes the TTC threshold to distinguish risky carfollowing situations from safe ones, which is set as 2 seconds in this study. Previous research found that the VSL control may increase travel time since relatively smaller speed limits are applied. In this study, the total travel time (TTT) is also applied to quantify the travel efficiency, which can be calculated as follows:

$$
\mathrm{TTT}=\sum_{i=1}^{M} T_{i}
$$

where $T_{i}$ is the travel time of the vehicle $i$.

\section{Simulation Results}

6.1. Simulation Experimental Design. The simulation experiments are conducted to test the performance of the proposed CAV-VSL system under different scenarios. The driver's sight distance $d$ is set to be $100 \mathrm{~m}$, which is the same as the distance from the VMS to the matched detector. The traffic flow is set to be $1200 \mathrm{veh} / \mathrm{hr} / \mathrm{ln}$, and the initial speed of each vehicle is set to be $30 \mathrm{~m} / \mathrm{s}$. All vehicles are expected to reduce the speed to $5 \mathrm{~m} / \mathrm{s}$ when reaching the bottleneck area. The leading vehicle brakes to the preset bottleneck speed and forms a shock wave propagating back upstream. For each experiment, the simulation of 1 hour is used for the comparison, with the first 5 minutes as the warm-up period. Other parameter settings are shown in Table 1 based on previous studies [37-41].

Note that applying homogenous IDM may have some limitations. In this study, however, we employed the same IDM for simulations due to some reasons: (1) if the homogenous IDM is not applied, we may simulate different human-driven vehicles with heterogenous model parameters calibrated by empirical data, but we cannot capture real heterogenous behaviors of CAVs due to the lack of data. In this case, the simulation result differences may result from different models instead of control strategies, which negatively affects the comparison of control performances; (2) the homogenous IDM has been extensively utilized in previous studies to describe both human-driven vehicles and CAVs [42, 43, 47]. 


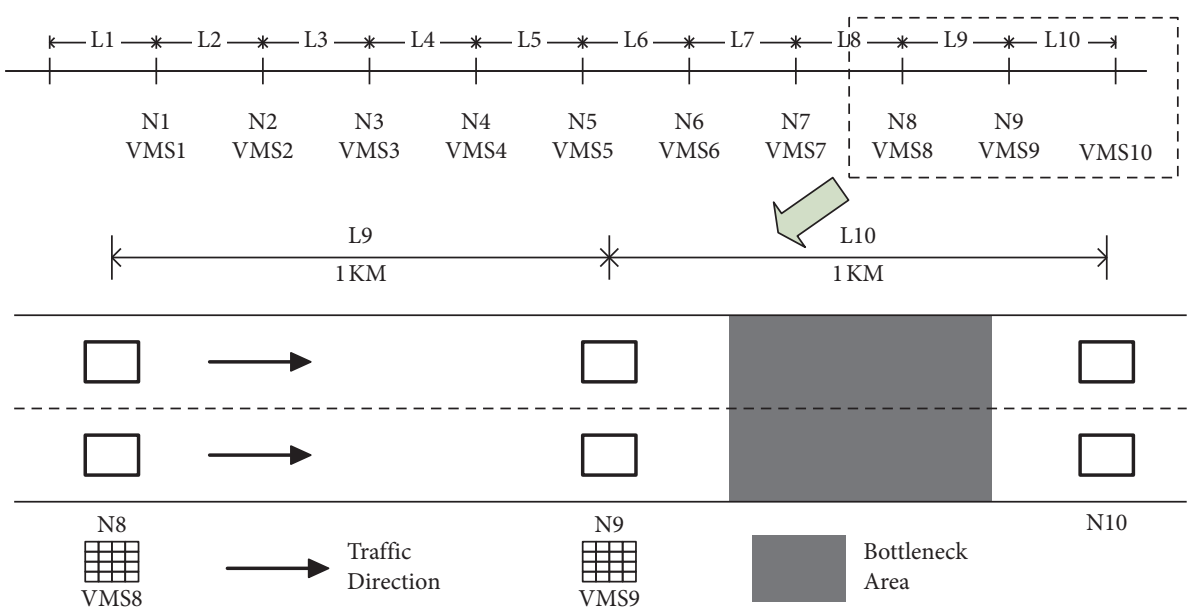

Figure 3: The simulation roadway with the bottleneck.

TABLE 1: Parameter settings of simulation experiments.

\begin{tabular}{|c|c|c|c|}
\hline \multirow{2}{*}{ Parameters } & \multirow{2}{*}{ Meaning } & \multicolumn{2}{|r|}{ Value } \\
\hline & & HDV & CAV \\
\hline$d(\mathrm{~m})$ & Sight distance & 100 & $100,(500,900)$ \\
\hline$\beta\left(\mathrm{m} / \mathrm{s}^{2}\right)$ & Desired deceleration & 2 & 2 \\
\hline$t_{a}(\mathrm{~s})$ & Perception reaction time & 1 & 0 \\
\hline $\bar{L}(\mathrm{~m})$ & Average vehicle length & 5 & 5 \\
\hline$\alpha_{m}\left(\mathrm{~m} / \mathrm{s}^{2}\right)$ & Maximum acceleration & 1 & 1 \\
\hline$v_{0}(\mathrm{~km} / \mathrm{h})$ & Desired speed & 120 & 120 \\
\hline$s_{0}(\mathrm{~m})$ & Minimum gap distance at standstill & 0 & 0 \\
\hline$T(\mathrm{~s})$ & Safe time gap & $1.1-1.6$ & $0.6,0.7,0.9,1.1(57 \%, 24 \%, 7 \%, 12 \%)$ \\
\hline$\Delta T(\mathrm{~s})$ & Data aggregation interval & 30 & 30 \\
\hline$\Delta t(\mathrm{~s})$ & Simulation time step & 0.1 & 0.1 \\
\hline
\end{tabular}

The execution process of the proposed CAV-VSL system is illustrated in Figure 4; the speed of all vehicles is reduced from $30 \mathrm{~m} / \mathrm{s}$ to $5 \mathrm{~m} / \mathrm{s}$ by gradual deceleration. Note that lanechanging behavior is not incorporated in this study for simplicity, as the major focus of this study is the reduction of rear-end collision risk.

The impacts of the VSL control and the CAV MPR on traffic safety were also explored in the simulation. The scenario with no VSL control and 0\% CAV MPR was simulated as the benchmark for comparison. Then, the simulation experiments of the influence of drivers' heterogeneity on the performance of the control system were conducted. Furthermore, the change of control execution site of CAV in the proposed CAV-VSL system was tested considering the advanced wireless communication technology, which makes up for the limitation of human drivers' sight distance. Finally, the simulation also explored the effect of CAVs on HDVs through the experiments of three different distributions of CAVs.

6.2. Results of the Safety Impact of the CAV-VSL System. The traffic flow consisting of all HDVs without any control strategies was firstly simulated as the base case. Then, different CAV MPRs and VSL control were tested and results of safety impacts are displayed in Figure 5. The horizontal axes are TET$^{*}$ and TIT $^{*}$, which are normalized results of TET and TIT and can be calculated as follows:

$$
\begin{aligned}
& \mathrm{TET}^{*}=\frac{\mathrm{TET}-\mathrm{TET}_{100 \%}^{\mathrm{VSL}}}{\mathrm{TET}_{0 \%}^{\mathrm{NO}}-\mathrm{TET}_{100 \%}^{\mathrm{VSL}},} \\
& \mathrm{TIT}^{*}=\frac{\mathrm{TIT}-\mathrm{TIT}_{100 \%}^{\mathrm{VSL}}}{\mathrm{TIT}_{0 \%}^{\mathrm{NO}}-\mathrm{TIT}_{100 \%}^{\mathrm{VSL}}},
\end{aligned}
$$

where the $\mathrm{TET}_{0 \%}^{\mathrm{NO}}$ is the TET of scenarios without CAVs and any control strategies and $\mathrm{TET}_{100 \%}^{\mathrm{VSL}}$ is the TET of scenarios with $100 \%$ CAV MPR and VSL control strategies.

Eleven different values of CAV MPRs were tested in the simulation, ranging from $0 \%$ to $100 \%$. When CAV MPR reaches $100 \%$, for both VSL control and no control scenarios, the results of $\mathrm{TET}_{100 \%}^{\mathrm{VSL}}$ and $\mathrm{TIT}_{100 \%}^{\mathrm{VSL}}$ are zero in simulations. In the scenario with MPR of $90 \%$ and without VSL control, TET* and TIT* are decreased by $55 \%$ (from $100 \%$ to $45 \%$ ) and $58 \%$ (from $100 \%$ to $42 \%$ ). When CAV MPR is $0 \%$, the VSL control achieves the reductions of $56 \%$ (from $100 \%$ to $44 \%$ ) and $59 \%$ (from $100 \%$ to $41 \%$ ) in TET $^{*}$ and TIT*, respectively. The above results indicate that both CAV and VSL have the potentials to improve traffic safety separately.

With the collaborative effect of the CAV-VSL system, the safety performance of VSL control is further improved whatever the MPR is. For example, when CAV MPR is $90 \%$, 


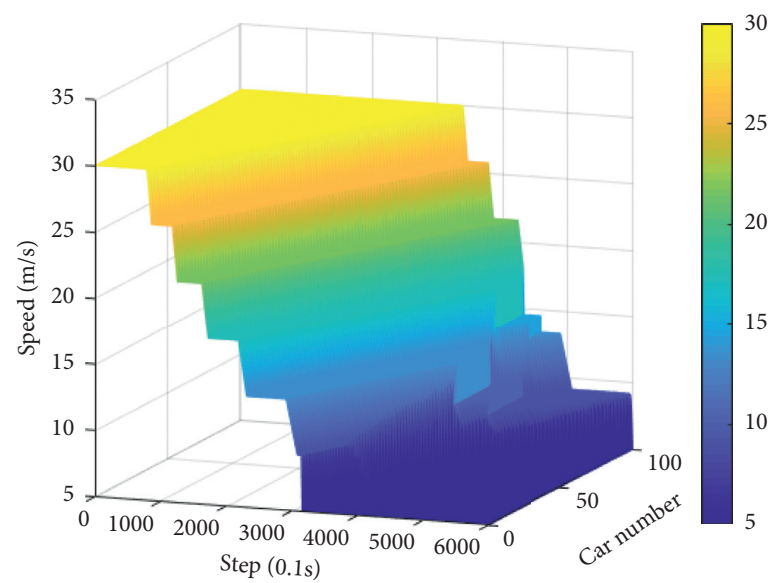

FIGURE 4: Illustration of the gradual deceleration process of the proposed CAV-VSL control.

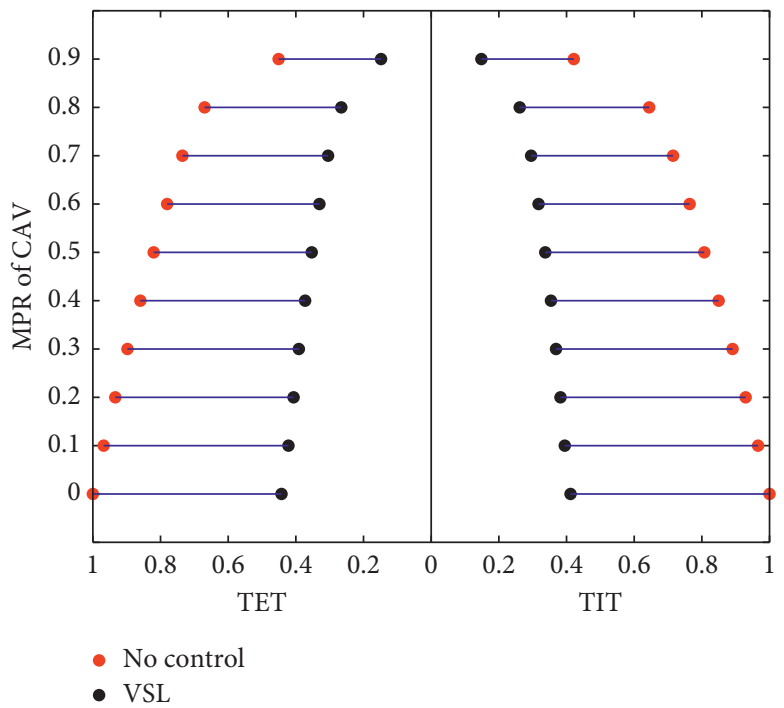

Figure 5: The safety impact of CAV-VSL control (normalization results).

both TET $^{*}$ and TIT $^{*}$ are reduced by $85 \%$ (from $100 \%$ to $15 \%$ ). It implies the great safety benefits of the proposed CAV-VSL system. When the control system calculates the suggested speed value according to the upstream and downstream traffic flow data, it will be delivered to CAVs accurately through advanced vehicle-to-infrastructure technology and guide CAVs to decelerate precisely and timely.

Note that the original TIT and TET values in scenarios with 100\% CAV MPR are found to be zero in simulation experiments, which means an absolutely safe condition. Nevertheless, it is impractical that all vehicles in the platoon have wireless communication functionality in the near future. The application and popularization of CAV still need a long term. Therefore, how to improve the safety performance of control systems under mixed flow is still a promising topic.

6.3. Results of the Impact of Driver Heterogeneity. The different responses of human drivers to VSL controls are the key factors restricting the control effectiveness. Figure 6 shows the influence of driver heterogeneity on the VSL control effect in terms of safety and efficiency under different CAV MPRs. As shown in Figures 6(a)-6(b), under different CAV MPRs, driver heterogeneity increases both TET and TIT values. For example, when CAV MPR is $0 \%$, with the noncompliance rate from $0 \%$ to $50 \%$, the TET and TIT rise by $42 \%$ (from 11,114 to 15,760 ) and $24 \%$ (from 20,139 to 24,877), respectively. While if CAV MPR is $90 \%$, the TET and TIT increase by $21 \%$ (from 3,724 to 4,501 ) and $20 \%$ (from 7,251 to 8,700), respectively.

Another interesting result is that under the scenario with $0 \%$ CAV MPR, noncompliance behavior has a greater impact on safety than overcompliance, while in the case of high CAV MPR, the influence is opposite. Specifically, when CAV MPR is $0 \%$, TET with the noncompliance rate of $50 \%$ is greater than it with overcompliance rate of $50 \%(15,760$ versus 14,746). Nevertheless, if CAV MPR is $90 \%$, the result is the opposite $(4,501$ versus 5,809$)$.

Given different heterogeneity rates, the increase of CAV MPR reduces the risk of rear-end collision. When the CAV 


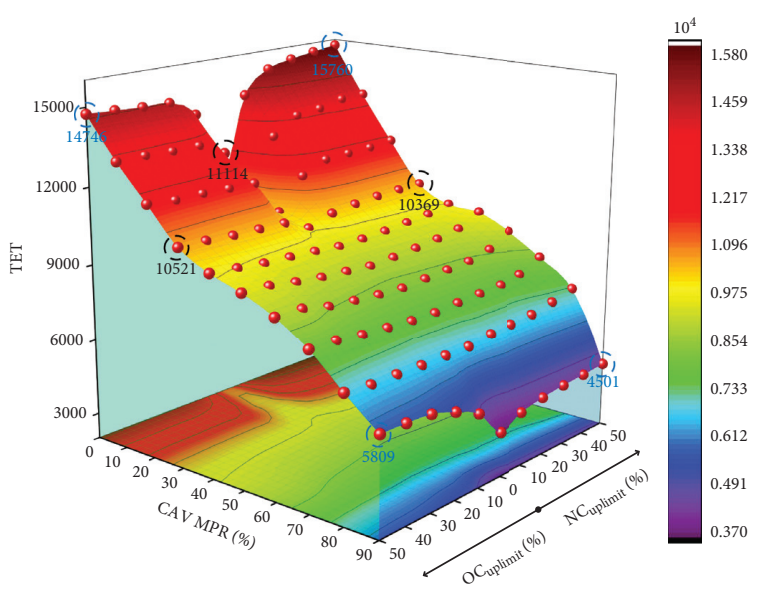

(a)

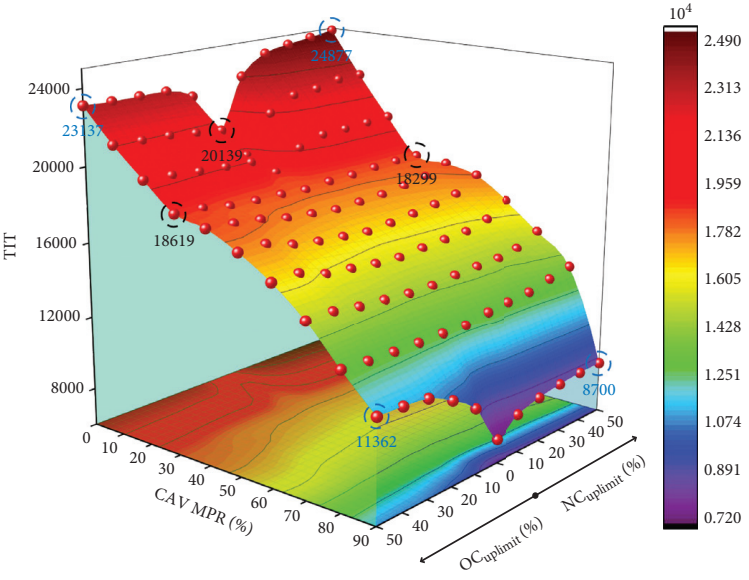

(b)

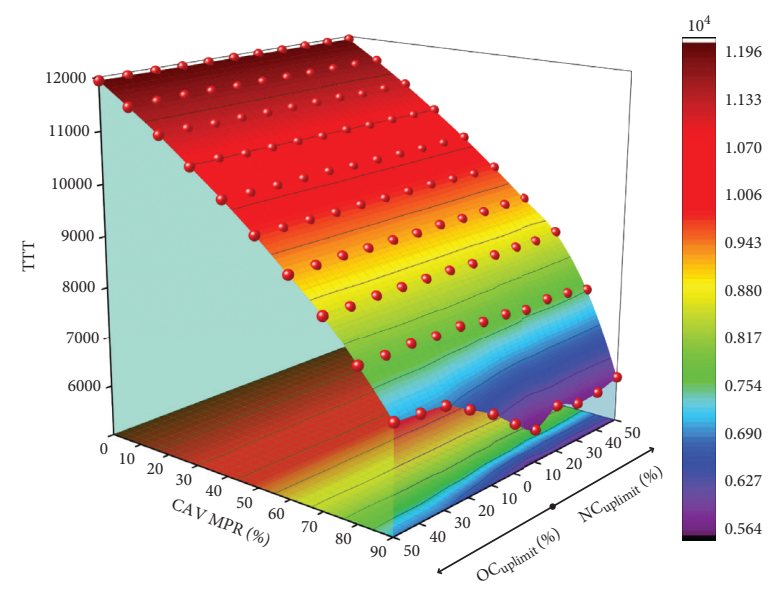

(c)

FIGURE 6: Impact of driver heterogeneity on safety and efficiency. (a) TET. (b) TIT. (c) TTT.

MRP reaches 30\% with heterogeneity, it can achieve a better effect than the scenario with 0\% CAV MRP without driver heterogeneity. For instance, the values of TET (the maximum value is 10,521 ) with over $30 \%$ CAV MPR are less than that 11,114 in the scenario of HDVs only without driver heterogeneity. It indicates that the CAVs can smooth the heterogeneity of human drivers and improve the safety of mixed flow by the proposed CAV-VSL control system.

Besides, as shown in Figure 6(c), driver heterogeneity has little influence on the total travel time. The possible reason may be that the heterogeneity rate has little change on the actual driving speed of drivers with the temporal and spatial constraints of the speed limit.

\subsection{Results of Impact of CAV Communication Range.} The vehicle-to-infrastructure communication technology makes it possible to transmit speed limit values to each CAV and remove another drivers' limitation, i.e., sight distance. The execution position of CAVs initiating deceleration can adjust flexibly with different communication ranges. The longer the execution distance of the control, the smaller the deceleration rate in the slowdown process. Generally, the communication range of vehicle-to-infrastructure can reach $1 \mathrm{~km}$ with WiMAX and $5 \mathrm{~km}$ with $5 \mathrm{~g}$ Cellular-V2X $[48,49]$. In this study, three execution sites within the communication range of vehicle-to-infrastructure are tested, which are short $(100 \mathrm{~m})$, medium $(500 \mathrm{~m})$, and long $(900 \mathrm{~m})$. Among them, the short one serves as the base case for execution sites comparison.

The simulation results are shown in Figure 7. It can be found that the safety performance of the CAV-VSL system improves as the communication range increases, except the MPR of $90 \%$. With the CAV MPR fewer than $50 \%$, the safety improvement of longer execution distance becomes more significant. When the CAV MPR reaches $90 \%$, the medium execution site works the best, indicating that the optimal control execution site under high CAV MPR is not consistent with that under low MPR. The possible reason may be that the long execution site causes the CAV to slow down early at the upstream detector, resulting in a premature deceleration behavior.

As shown in Tables 2 and 3, the sensitivity analysis considering compliance issues is further conducted. Table 2 describes the proportion of safety improvement for the medium execution site relative to the short execution 


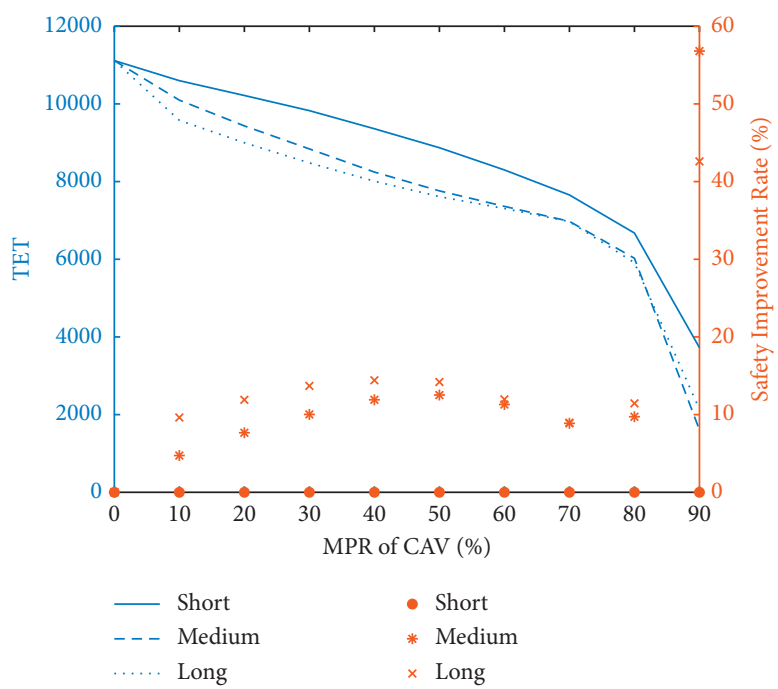

(a)

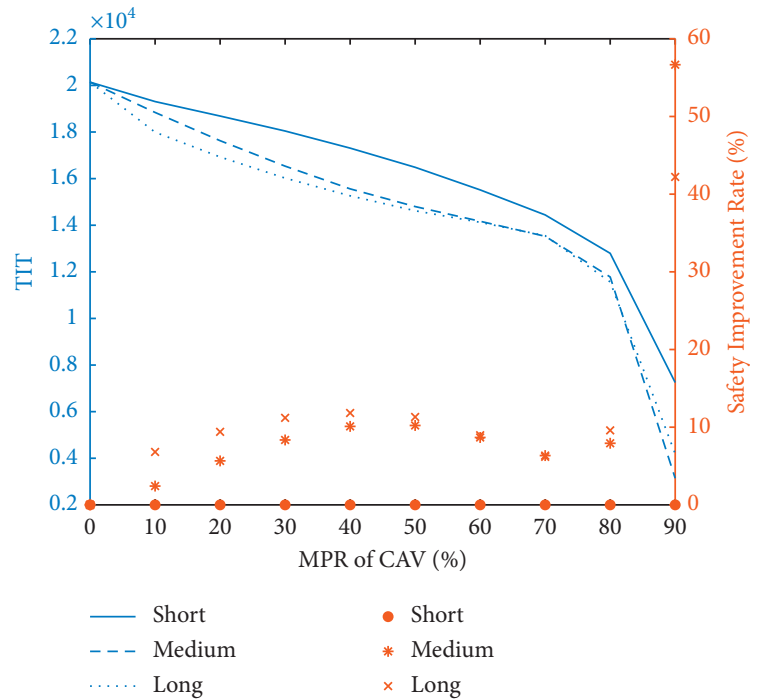

(b)

FIgURE 7: Results of three control execution sites. (a) TET. (b) TIT.

TABLE 2: Safety improvement rates (medium vs. short).

\begin{tabular}{|c|c|c|c|c|c|c|c|c|c|c|c|c|c|}
\hline & & \multicolumn{6}{|c|}{$\mathrm{NC}_{\text {uplimit }}(\%)$} & \multicolumn{6}{|c|}{ OC $_{\text {uplimit }}(\%)$} \\
\hline & & 0 & 10 & 20 & 30 & 40 & 50 & 0 & 10 & 20 & 30 & 40 & 50 \\
\hline \multicolumn{14}{|l|}{ TET } \\
\hline \multirow{9}{*}{ MPR of CAV } & $10 \%$ & 4.73 & 8.12 & 7.74 & 7.72 & 7.84 & 6.95 & 4.73 & 4.38 & 3.08 & 3.18 & 1.72 & 2.34 \\
\hline & $20 \%$ & 0.66 & 11.25 & 12.03 & 10.80 & 10.53 & 10.49 & 7.66 & 9.66 & 6.09 & 4.62 & 3.66 & 3.27 \\
\hline & $30 \%$ & 0.03 & 10.86 & 11.55 & 11.48 & 10.71 & 10.12 & 10.03 & 10.39 & 6.67 & 4.57 & 3.75 & 4.25 \\
\hline & $40 \%$ & 11.91 & 10.02 & 8.79 & 7.98 & 7.52 & 7.46 & 11.91 & 10.66 & 6.20 & 3.45 & 2.50 & 3.07 \\
\hline & $50 \%$ & 12.52 & 8.20 & 6.83 & 6.05 & 5.16 & 4.95 & 12.52 & 10.45 & 5.19 & 2.22 & 1.49 & 1.70 \\
\hline & $60 \%$ & 1.30 & 6.73 & 4.45 & 3.85 & 3.43 & 2.81 & 11.30 & 9.31 & 3.79 & 0.37 & 0.84 & 0.45 \\
\hline & $70 \%$ & 8.88 & 4.22 & 2.86 & 2.78 & 1.14 & 1.91 & 8.88 & 7.02 & 2.98 & 0.87 & 0.78 & 0.56 \\
\hline & $80 \%$ & 9.73 & 7.22 & 7.74 & 6.38 & 2.46 & 1.81 & 9.73 & 7.87 & 2.20 & 0.33 & 0.60 & 1.87 \\
\hline & $90 \%$ & 56.80 & 18.38 & 34.36 & 21.59 & 25.05 & 35.01 & 56.80 & 34.72 & 26.21 & 27.49 & 17.71 & 7.83 \\
\hline \multicolumn{14}{|l|}{ TIT } \\
\hline \multirow{9}{*}{ MPR of CAV } & $10 \%$ & 2.42 & 4.40 & 4.50 & 4.44 & 5.00 & 4.45 & 2.42 & 1.65 & 1.07 & 1.39 & 0.65 & 1.13 \\
\hline & $20 \%$ & 5.66 & 7.17 & 7.59 & 6.83 & 6.90 & 6.78 & 5.66 & 5.72 & 3.16 & 2.29 & 1.78 & 1.94 \\
\hline & $30 \%$ & 8.36 & 7.96 & 8.04 & 7.91 & 7.30 & 6.85 & 8.36 & 7.13 & 3.84 & 2.29 & 1.73 & 2.28 \\
\hline & $40 \%$ & 10.10 & 7.84 & 6.54 & 5.76 & 5.41 & 5.27 & 10.10 & 8.14 & 4.01 & 1.67 & 0.74 & 1.37 \\
\hline & $50 \%$ & 10.22 & 6.27 & 5.06 & 4.36 & 3.55 & 3.26 & 10.22 & 7.95 & 3.59 & 1.05 & 0.28 & 0.54 \\
\hline & $60 \%$ & 8.68 & 4.96 & 3.04 & 2.61 & 2.32 & 1.81 & 8.68 & 6.81 & 2.50 & 0.05 & 1.14 & 0.06 \\
\hline & $70 \%$ & 6.35 & 2.91 & 1.96 & 2.05 & 0.54 & 1.36 & 6.35 & 4.52 & 1.92 & 0.64 & 0.78 & 0.20 \\
\hline & $80 \%$ & 7.93 & 6.59 & 7.34 & 6.05 & 2.16 & 1.67 & 7.93 & 5.94 & 1.36 & 0.15 & 0.52 & 1.49 \\
\hline & $90 \%$ & 6.66 & 18.17 & 34.48 & 21.75 & 25.14 & 35.20 & 56.66 & 34.27 & 26.04 & 27.48 & 17.81 & 7.67 \\
\hline
\end{tabular}

site, and Table 3 displays the proportion of safety improvement for the long execution site relative to the medium one. The medium and long strategy execution sites bring more safety benefits than the short execution site under different driver heterogeneity levels. In the case of low CAV MPR, the long strategy execution site is safer than the medium one, but in the case of high CAV MPR, the result is also the opposite.

6.5. Results of the Impact of CAV Position Distribution. Compared to HDVs, CAVs can more accurately receive speed limit information and be more compliant and further enforce driving behaviors of the following vehicles. When the leading CAV slows down, the following HDVs will be forced to decelerate to keep a safe distance. In order to explore the effect of CAV position distribution on safety improvement, the following experiments were conducted with $\mathrm{NC}_{\text {uplimit }}$ and $\mathrm{OC}_{\text {uplimit }}$ both equal to $20 \%$ with long execution site. In the mixed traffic flow, three types of distributions are tested, including front aggregation, rear aggregation, and uniform distribution, and results are shown in Figure 8. In the case of low MPR, the TET and TIT indexes are minimum when CAVs are uniformly distributed. With respect to high MPR, however, front aggregation obtains the most significant safety improvement. 
TABLE 3: Safety improvement rates (long versus medium).

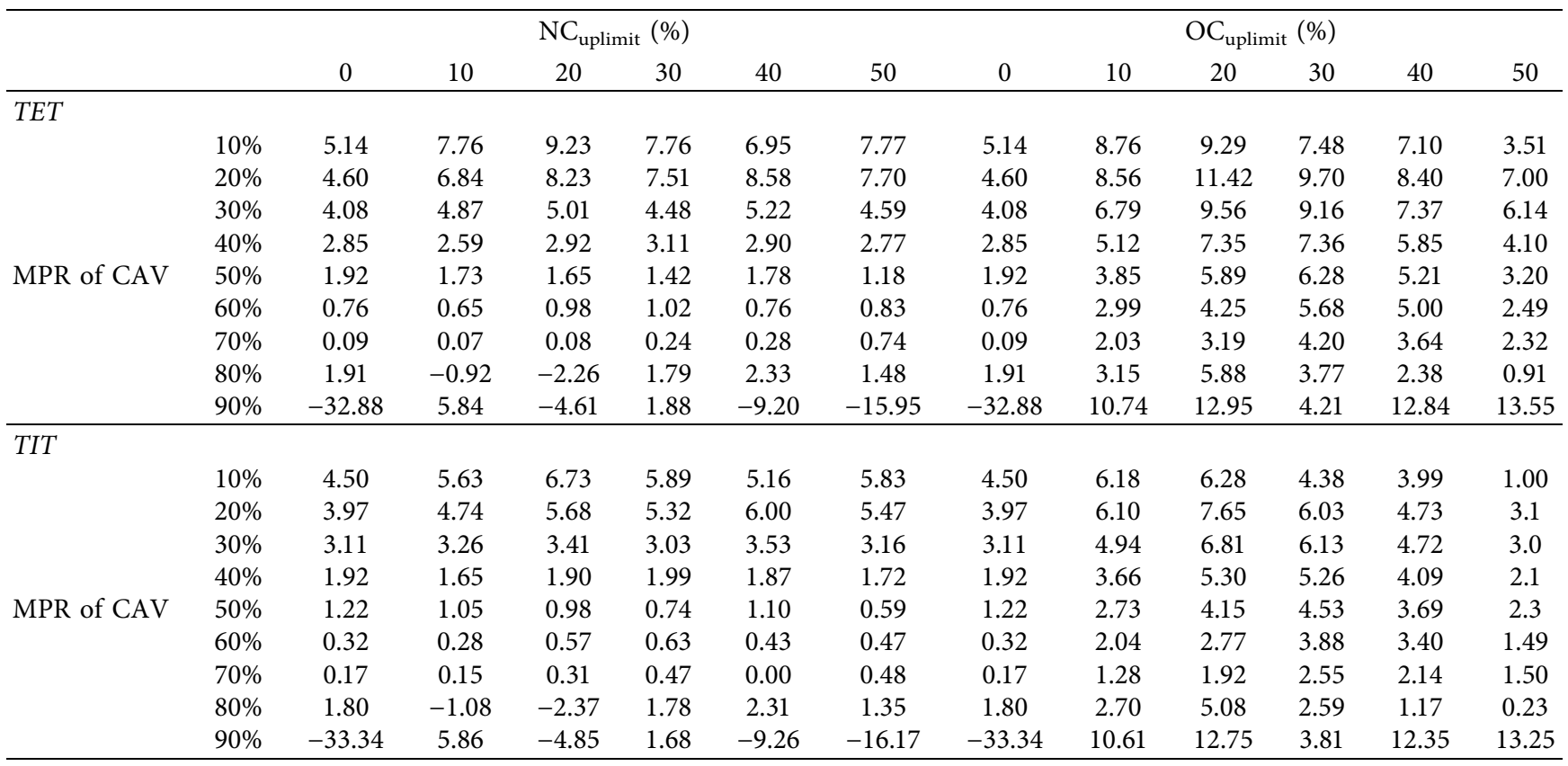
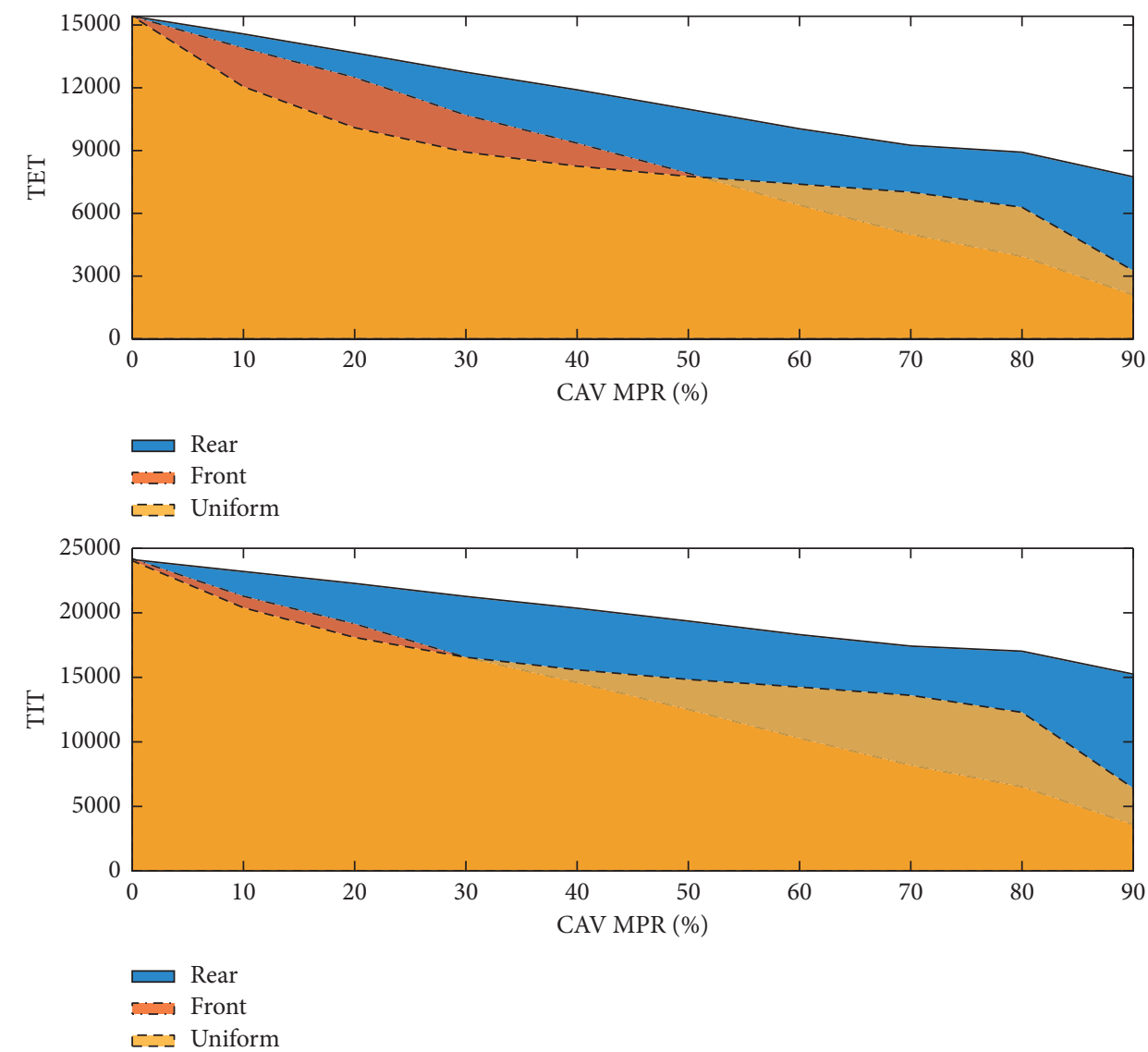

FIgURE 8: The impact of the position distribution of CAVs.

The core of the comparison among the three distributions is actually the interaction impact between the aggregation effect and the guidance effect of CAVs. The aggregation effect represents the system benefit brought by
CAV aggregation, and the guidance effect represents the system benefit brought by the influence of leading CAVs on following HDVs' driving behaviors. Obviously, rear aggregation maximizes the aggregation effect but has no guidance 


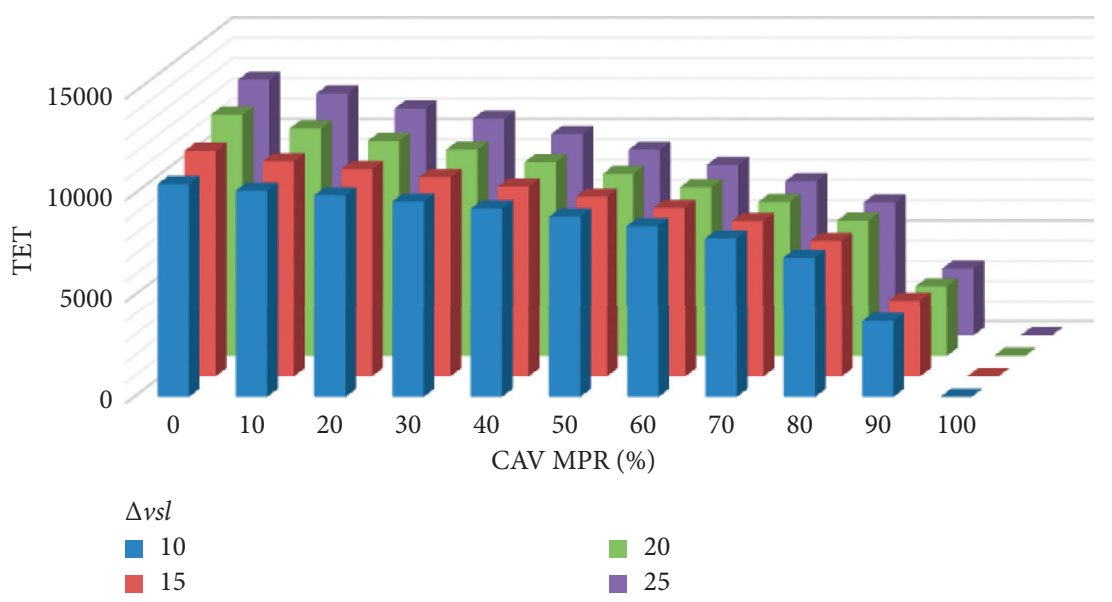

(a)

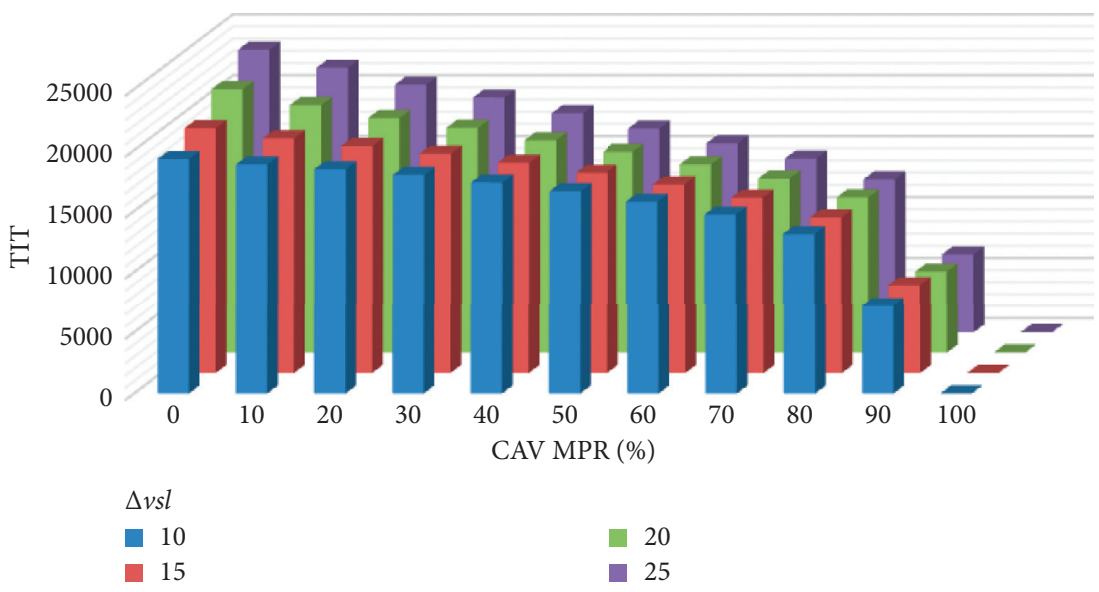

(b)

FIgure 9: The results of sensitivity analysis of $\Delta_{v s l}$ (a) TET. (b) TIT.

effect. On the contrary, uniform distribution maximizes the guidance effect, while there is no aggregation effect. The front aggregation maximizes the guidance effect and has the guidance effect at the junction of CAVs and HDVs clusters to some extent. In the case of low MPR, the optimal system safety benefits can be obtained under the uniform distribution of CAVs, because the guidance effect on HDVs could alleviate the adverse effects of driver heterogeneity.

6.6. Results of Sensitivity Analysis of $\Delta_{v s l}$. As for the variable speed limit control strategy, the selection of hyperparameter will also have an effect on the experimental results. To explore the impact of hyperparameter on safety improvement, four different values of $\Delta_{v s l}$ were tested in the simulation, ranging from $10 \mathrm{~km} / \mathrm{h}$ to $25 \mathrm{~km} / \mathrm{h}$ with an interval of $5 \mathrm{~km} / \mathrm{h}$, and the results are displayed in Figure 9. In the scenario with CAV MPR of $0 \%$, the two surrogate safety measures increase with the rise of $\Delta_{v s l}$, where TET and TIT are both increased by about $20 \%$ (TET: from 10,481 to 12,619 , TIT: from 19,284 to 23,234). As the increase of CAV MPR, the increasing effect gradually weakens. In the scenario with CAV MPR of $50 \%$, with the increase of $\Delta_{v s l}$, TET and TIT increased by only $3 \%$ (from 8,883 to 9,175 ) and $1 \%$ (from 16,640 to 16,760 ), respectively. When CAV MPR reaches more than $50 \%$, the increase of $\Delta_{v s l}$ has few impacts on safety. The above results indicate that, in the early stage of mixed flow, selecting appropriate $\Delta_{v s l}$ will further improve the effect of variable speed limit control. Furthermore, the results also imply the necessity of spatial-temporal constraints.

\section{Conclusions and Recommendations}

This study proposed a CAV-VSL control system to reduce the risk of rear-end collision near a bottleneck on a freeway. Firstly, human driver heterogeneity, which may reduce the control effect of VSL controls, was discussed. Then, the CAV-VSL control system was proposed to eliminate the limitations arising from driver heterogeneity, and extensive simulation experiments were conducted in different scenarios. According to the experimental results, the following conclusions are drawn:

(1) Both CAV and VSL have positive effects on traffic safety improvement in the mixed traffic flow separately. The proposed CAV-VSL control system can better perform by the collaborative effect of CAV and VSL control. 
(2) Heterogeneity of human drivers, including the noncompliance and overcompliance to the speed limit as well as sight distance, has negative impacts on the control performance of VSL.

(3) CAVs can overcome the limitation of the VSL control effect caused by human drivers' heterogeneity. The advanced communication technology of CAVs could improve the safety performance of the proposed CAV-VSL control system by adjusting the deceleration execution site. CAVs can accurately execute the control requirement and homogenize the response of following HDVs to the recommended speed limit.

(4) The influence of CAVs' position distribution has a significant effect. The front or rear aggregation distributions bring benefits via pure CAV platoons, while the uniform distribution brings benefits for safety by the impact of leading CAVs on following HDVs' driving behaviors. The different position distributions are suitable for different CAV MPRs.

(5) The increase of hyperparameter $\Delta_{v s l}$ has a negative impact on the safety performance of the control system, which is due to the relaxation of spatialtemporal constraints.

The mixed flow of CAVs and HDVs is expected to exist for a long time, and it is urgent to study the proactive traffic control strategy under different CAV MPRs. The proposed CAV-VSL system is effective in reducing the influence of the heterogeneity of the human drivers and obtaining a much better VSL control performance in the mixed traffic flow. However, there are still many issues that need to be addressed. First, given the low CAV MPR, how to optimally deploy the limited number of CAVs to different lanes at different positions to achieve uniform distribution and maximize safety benefits should be explored. Besides, in the case of a higher CAV MPR, the control execution sites have a significant impact on traffic safety. The optimal position for CAVs to decelerate is required to be investigated as well. Meanwhile, the distribution of obeyance may be calibrated with empirical data in the future and the potential stochastic optimal strategy may be obtained with the calibrated distribution, which is also worthy of investigation. Besides, the car-following model and reaction pattern can be different between human-driven vehicles and CAVs, which needs to be further explored in future research. These topics should be addressed in follow-up studies.

\section{Data Availability}

The data used to support the findings of this study are available from the corresponding author upon request.

\section{Conflicts of Interest}

The authors declare that they have no conflicts of interest.

\section{Acknowledgments}

This research was supported by the Fundamental Research Funds for the Central Universities, CHD (No. 300102341502), the National Natural Science Foundation of China (No. 71901223), the Foundation of Central South University (No. 202045010), Innovation-Driven Project of Central South University (No. 2020CX013), and Central South University Postgraduate Independent Exploration and Innovation Project (No. 2021zzts0693).

\section{References}

[1] S. Li, T. Wang, H. Ren et al., "Variable speed limit strategies based on the macro hierarchical control traffic flow model," Journal of Advanced Transportation, vol. 2021, 2021.

[2] X. Qu, L. Li, Z. Yi, P. Mao, and M. Yang, "Traffic flow modeling of freeway variable speed limit control based on the big data of driving behavior," Journal of Advanced Transportation, vol. 2020, 2020.

[3] X. Qu, M. Yang, J. Ji, L. Li, and B. Ran, “Analyzing the safety impacts of variable speed limit control on aggregated driving behavior based on traffic big data," Journal of Advanced Transportation, vol. 2021, 2021.

[4] P. Wang, Y. Zhang, S. Wang, L. Li, and X. Li, "Forecasting travel speed in the rainfall days to develop suitable variable speed limits control strategy for less driving risk," Journal of Advanced Transportation, vol. 2021, 2021.

[5] W. Wang and Z. Cheng, "Variable speed limit signs: control and setting locations in freeway work zones," Journal of Advanced Transportation, vol. 2017, p. 4390630, 2017.

[6] M. Kiec, C. D’Agostino, and S. Pazdan, "Impact on road safety and operation of rerouting traffic in rural travel time information system," Sensors, vol. 20, no. 15, 2020.

[7] S. Li, T. Wang, H. Ren, B. Shi, and X. Kong, "Optimization model and method of variable speed limit for urban expressway," Journal of Advanced Transportation, vol. 2021, 2021.

[8] X. Wang, R. Zhang, Y. Gou, J. Liu, L. Zhao, and Y. Li, "Variable speed limit control method of freeway mainline in intelligent connected environment," Journal of Advanced Transportation, vol. 2021, 2021.

[9] M. Saad, M. Abdel-Aty, J. Lee, and L. Wang, "Safety analysis of access zone design for managed toll lanes on freeways," Journal of Transportation Engineering Part A-Systems, vol. 144, no. 11, 2018.

[10] L. Wu, Y. Ci, Y. Sun, and W. Qi, "Research on joint control of on-ramp metering and mainline speed guidance in the urban expressway based on mpc and connected vehicles," Journal of Advanced Transportation, vol. 2020, 2020.

[11] Y. Zhou, K. Ozbay, P. Kachroo, and F. Zuo, "Ramp metering for a distant downstream bottleneck using reinforcement learning with value function approximation," Journal of Advanced Transportation, vol. 2020, 2020.

[12] Z. Zhibin Li, L. Pan Liu, W. Wei Wang, and C. Chengcheng $\mathrm{Xu}$, "Development of a control strategy of variable speed limits to reduce rear-end collision risks near freeway recurrent bottlenecks," IEEE Transactions on Intelligent Transportation Systems, vol. 15, no. 2, pp. 866-877, 2014.

[13] M. Abdel-Aty, J. Dilmore, and A. Dhindsa, "Evaluation of variable speed limits for real-time freeway safety improvement," Accident Analysis \& Prevention, vol. 38, no. 2, pp. 335-345, 2006. 
[14] C. Lee, B. Hellinga, and F. Saccomanno, "Evaluation of variable speed limits to improve traffic safety," Transportation Research Part C: Emerging Technologies, vol. 14, no. 3, pp. 213-228, 2006.

[15] R. A. Boateng, M. Fontaine, and Z. H. Khattak, "Driver response to variable speed limits on I-66 in northern Virginia," Journal of Transportation Engineering, vol. 145, no. 6, pp. 4019021-4019029, 2019.

[16] J. R. Frejo, I. Papamichail, M. Papageorgiou, and B. De Schutter, "Macroscopic modeling of variable speed limits on freeways," Transportation Research Part C: Emerging Technologies, vol. 100, pp. 15-33, 2019.

[17] Y. Wu, M. Abdel-Aty, L. Wang, and M. S. Rahman, "Combined connected vehicles and variable speed limit strategies to reduce rear-end crash risk under fog conditions," Journal of Intelligent Transportation Systems, vol. 24, no. 5, pp. 494-513, 2020.

[18] Y. Han, D. Chen, and S. Ahn, "Variable speed limit control at fixed freeway bottlenecks using connected vehicles," Transportation Research Part B: Methodological, vol. 98, pp. 113-134, 2017.

[19] L. Ye, C. Xu, X. Lu, and W. Wei, "Integrated cooperative adaptive cruise and variable speed limit controls for reducing rear-end collision risks near freeway bottlenecks based on micro-simulations," IEEE Transactions on Intelligent Transportation Systems, vol. 18, no. 11, pp. 3157-3167, 2017.

[20] X. Zhao, W. Xu, J. Ma, H. Li, Y. Chen, and J. Rong, "Effects of connected vehicle-based variable speed limit under different foggy conditions based on simulated driving," Accident Analysis \& Prevention, vol. 128, pp. 206-216, 2019.

[21] R. C. Carlson, I. Papamichail, and M. Papageorgiou, "Integrated feedback ramp metering and mainstream traffic flow control on motorways using variable speed limits," Transportation Research Part C: Emerging Technologies, vol. 46, pp. 209-221, 2014.

[22] G. R. Iordanidou, C. Roncoli, I. Papamichail, and M. Papageorgiou, "Feedback-based mainstream traffic flow control for multiple bottlenecks on motorways," IEEE Transactions on Intelligent Transportation Systems, vol. 16, no. 2, pp. 610-621, 2015.

[23] Z. Li, C. Xu, Z. Pu, Y. Guo, and P. Liu, "Reinforcement learning-based variable speed limits control to reduce crash risks near traffic oscillations on freeways," IEEE Intelligent Transportation Systems Magazine, vol. 13, no. 4, pp. 64-70, 2020.

[24] A. Hegyi, B. D. Schutter, and J. Hellendoorn, "Optimal coordination of variable speed limits to suppress shock waves," in Proceedings of the IEEE Conference on Decision \& Control, IEEE, Maui, HI, USA, Dec. 2003.

[25] Y. Wang and P. A. Ioannou, "New model for variable speed limits," Transportation Research Record: Journal of the Transportation Research Board, vol. 2249, no. 1, pp. 38-43, 2011.

[26] M. Hadiuzzaman, "Cell transmission model based variable speed limit control for freeway," in Proceedings of the TRB 91st Annual Meeting Compendium of Papers, Transportation Research Board, Washington, D.C., January 2012.

[27] Z. Li, P. Liu, C. Xu, and W. Wang, "Optimal mainline variable speed limit control to improve safety on large-scale freeway segments," Computer-Aided Civil and Infrastructure Engineering, vol. 31, no. 5, pp. 366-380, 2016.

[28] P. Edara, C. Sun, and Y. Hou, "Evaluation of variable advisory speed limits in congested work zones," Journal of Transportation Safety \& Security, vol. 9, no. 2, pp. 123-145, 2017.
[29] N. J. Fudala and M. D. Fontaine, "Interaction between system design and operations of variable speed limit systems in work zones," Transportation Research Record: Journal of the Transportation Research Board, vol. 2169, no. 1, pp. 1-10, 2010.

[30] Lu Xiao-Yun, E. Steven, and Shladover, "Review of variable speed limits and advisories: theory, algorithms, and practice," Transportation Research Record, vol. 2423, no. 1, pp. 15-23, 2018.

[31] Z. Li, Y. Li, P. Liu, W. Wang, and C. Xu, "Development of a variable speed limit strategy to reduce secondary collision risks during inclement weathers," Accident Analysis \& Prevention, vol. 72, pp. 134-145, 2014.

[32] Y. Wu, M. Abdel-A Ty, W. Ling, and S. Rahman, "Improving flow and safety in low visibility conditions by applying connected vehicles and variable speed limits technologies," in Proceedings of the Transportation Research Board 98th Annual Meeting, Transportation Research Board, Washington D.C., January 2019.

[33] B. Hellinga and M. Mandelzys, "Impact of driver compliance on the safety and operational impacts of freeway variable speed limit systems," Journal of Transportation Engineering, vol. 137, no. 4, pp. 260-268, 2011.

[34] N. J. Garber and A. A. Ehrhart, "Effect of speed, flow, and geometric characteristics on crash frequency for two-lane highways," Transportation Research Record: Journal of the Transportation Research Board, vol. 1717, no. 1, pp. 76-83, 2000.

[35] N. J. Garber and R. Gadiraju, "Factors affecting speed variance and its influence on accidents," Transportation Research Record Journal of the Transportation Research Board, vol. 1213, 1989.

[36] T. F. Golob, W. W. Recker, and V. M. Alvarez, "Freeway safety as a function of traffic flow," Accident Analysis \& Prevention, vol. 36, no. 6, pp. 933-946, 2004.

[37] Y. Li, Z. Li, H. Wang, W. Wang, and L. Xing, "Evaluating the safety impact of adaptive cruise control in traffic oscillations on freeways," Accident Analysis \& Prevention, vol. 104, pp. 137-145, 2017.

[38] Y. Li, H. Wang, W. Wang, S. Liu, and Y. Xiang, "Reducing the risk of rear-end collisions with infrastructure-to-vehicle (I2V) integration of variable speed limit control and adaptive cruise control system," Traffic Injury Prevention, vol. 17, no. 6, pp. 597-603, 2016.

[39] V. Milanés and S. E. Shladover, "Modeling cooperative and autonomous adaptive cruise control dynamic responses using experimental data," Transportation Research Part C: Emerging Technologies, vol. 48, pp. 285-300, 2014.

[40] M. Treiber, A. Hennecke, and D. Helbing, "Congested traffic states in empirical observations and microscopic simulations," Physical Review, vol. 62, no. 2, pp. 1805-1824, 2000.

[41] L. Xiao, M. Wang, W. Schakel, and B. van Arem, "Unravelling effects of cooperative adaptive cruise control deactivation on traffic flow characteristics at merging bottlenecks," Transportation Research Part C: Emerging Technologies, vol. 96, pp. 380-397, 2018.

[42] A. Kesting, M. Treiber, M. Schönhof, and D. Helbing, "Adaptive cruise control design for active congestion avoidance," Transportation Research Part C: Emerging Technologies, vol. 16, no. 6, pp. 668-683, 2008.

[43] A. Kesting, M. Treiber, M. Schönhof, F. Kranke, and D. Helbing, "Jam-avoiding adaptive cruise control (acc) and its impact on traffic dynamics," Traffic and Granular Flow'05, pp. 633-643, 2006. 
[44] Z. Li, W. Li, S. Xu, and Y. Qian, "Stability analysis of an extended intelligent driver model and its simulations under open boundary condition," Physica A: Statistical Mechanics and Its Applications, vol. 419, pp. 526-536, 2015.

[45] L. Ye, W. Hao, W. Wei, X. Lu, and X. Wei, "Evaluation of the impacts of cooperative adaptive cruise control on reducing rear-end collision risks on freeways," Accident Analysis \& Prevention, vol. 98, pp. 87-95, 2017.

[46] S. M. S. Mahmud and L. M. S. Hoque, "Application of proximal surrogate indicators for safety evaluation: a review of recent developments and research needs," IATSS Research, vol. 41, no. 4, pp. 153-163, 2017.

[47] A. Talebpour and H. S. Mahmassani, "Influence of connected and autonomous vehicles on traffic flow stability and throughput," Transportation Research Part C: Emerging Technologies, vol. 71, pp. 143-163, 2016.

[48] M. N. Ahangar, Q. Z. Ahmed, F. A. Khan, and M. Hafeez, "A survey of autonomous vehicles: enabling communication technologies and challenges," Sensors, vol. 21, no. 3, p. 706, 2021.

[49] C. M. Chou, C. Y. Li, W. M. Chien, and K. C. Lan, "A feasibility study on vehicle-to-infrastructure communication: wifi vs. Wimax," in Proceedings of the Tenth International Conference on Mobile Data Management: Systems, IEEE, Taipei, Taiwan, May 2009. 